\title{
Superhydrophobic Particles Derived from Nature-Inspired Polyphenol Chemistry for Liquid Marble Formation and Oil Spills Treatment
}

Shouying Huang, ${ }^{a}$ Yan Zhang, ${ }^{a}$ Jiafu Shi ${ }^{b,}{ }^{*}$ and Weiping Huang ${ }^{a, *}$

${ }^{a}$ College of Chemistry, Nankai University, Tianjin 300071, China.

${ }^{\mathrm{b}}$ School of Environmental Science and Engineering, Tianjin University, 300072

Tianjin, China.

*Corresponding author: Jiafu Shi, E-mail: shijiafu@tju.edu.cn; Weiping Huang, hwp914@nankai.edu.cn. 


\section{EXPERIMENTAL}

Materials: Tris(hydroxymethyl) aminomethane, tannic acid, silver nitrate, $\mathrm{HCl}$, ethanol and $1 \mathrm{H}, 1 \mathrm{H}, 2 \mathrm{H}, 2 \mathrm{H}-\mathrm{Perfluorodecanethiol}(97 \%)$ were purchased from Sigma-Aldrich Chem. Co. Titania particles were synthesized according to our previous work. ${ }^{1}$ All of the chemicals were used as received without further purification. The water used in the experiments is purified by a three-stage Millipore Milli-Q Plus system with a resistivity higher than $15.0 \mathrm{M} \Omega \mathrm{cm}$.

Fabrication of superhydrophobic titania/polyphenol/Ag particles: $50 \mathrm{mg}$ of titania particles were immersed into $2.0 \mathrm{mg} / \mathrm{mL}$ solutions of tannic acid in $30 \mathrm{~mL}$ buffered saline (50 mM Tris-HCl, $\mathrm{pH} 7.8$ ) for $30 \mathrm{~min}$ at room temperature with mild agitation. Tannic acid-modified particles were further sonicated in DI water for $10 \mathrm{sec}$, followed by rinsing with DI water and drying with nitrogen gas. Then, the particles were washed by de-ionized water for several times and dispersed in a $20 \mathrm{~mL} 1 \mathrm{mg} / \mathrm{mL}$ $\mathrm{AgNO}_{3}$ solution to react for $2 \mathrm{~h}$. After washing by water and ethanol, the composite particles were dispersed in $1 \mathrm{H}, 1 \mathrm{H}, 2 \mathrm{H}, 2 \mathrm{H}$-perfluorodecanethiol ethanolic solution $(1: 1000 \mathrm{v}: \mathrm{v})$ to react at room temperature overnight.

Fabrication of particle-functionalized sponges: $50 \mathrm{mg}$ of titania particles with two pieces of melamine sponges $\left(0.5 \mathrm{~cm}^{*} 2 \mathrm{~cm} * 2 \mathrm{~cm}\right)$ were immersed into $2.0 \mathrm{mg} / \mathrm{mL}$ solutions of tannic acid in $30 \mathrm{~mL}$ buffered saline $(50 \mathrm{mM}$ Tris- $\mathrm{HCl}, \mathrm{pH} 7.8)$ for 30 min at room temperature with mild agitation. The obtained sponges were rinsed with DI water and dried with nitrogen gas. Then, the sponges were washed by de-ionized water for several times and dispersed in a $20 \mathrm{~mL} 1 \mathrm{mg} / \mathrm{mL} \mathrm{AgNO}_{3}$ solution to react for $2 \mathrm{~h}$. After washing by water and ethanol, the sponges were dispersed in $1 \mathrm{H}, 1 \mathrm{H}, 2 \mathrm{H}, 2 \mathrm{H}-$ perfluorodecanethiol ethanolic solution $(1: 1000 \mathrm{v}: \mathrm{v})$ to react at room temperature overnight. 
Characterizations: Scanning electron microscopy (SEM, Nanosem 430) and the attached energy dispersive spectroscope (EDS) were performed to measure the morphology, elemental composition of the particles. X-ray photoelectron spectra (XPS) were recorded on a PHI 5000C ESCA System. High-resolution transmission electron microscopy (HRTEM) was performed on a (JEM-100CX II) instrument. The contact angles to water were determined by using a digital optical contact angle meter JC-2000C1 (Shanghai, China). The size distribution of the superhydrophobic particles was measured by a dynamic light scattering (DLS) system (Brookhaven Instruments BI200SM).

Absorption tests of oils/organic solvents: A series of oils/organic solvents (including crude oil, pump oil, used pump oil, methanol, ethanol, isopropyl alcohol, soybean oil, n-hexane, n-heptane, n-hexadecane, toluene, acetone, dichloromethane, chlorform, $D M F, D M S O$ ) were adopted in the typical absorption process. Briefly, the absorbent was dropped into the organic liquid until the absorbent was completely filled with the liquid. Then, the sponge was taken out and rapidly weighed.

The absorption capacity was defined as $\left(M_{a}-M_{0}\right) / M_{0}$, which was mainly applied for calculating the absorption abilities of the superhydrophobic sponge. $M_{0}$ was the weight of the pristine dry absorbent for absorbing organic liquid; $M_{a}$ was the total weight after saturation with liquids.

The net absorption capacity was defined as $\left(M_{n}-M_{n}\right.$, desorption $) / M_{0}$, which was mainly applied for calculating the recycling abilities of the superhydrophobic sponge. $M_{0}$ was the weight of the pristine dry absorbent for absorbing organic liquid; $M_{n}$ was the total weight after saturation with liquids at the $n^{\text {th }}$-time recycling, $M_{n \text {, desorption }}$ was the total weight after desorption of liquids at the $n^{\text {th }}$-time recycling. 
1 H. Wu, Y. Liang, J. Shi, X. Wang, D. Yang and Z. Jiang, Mater. Sci. Eng. C, 2013, 33, $1438-1445$. 

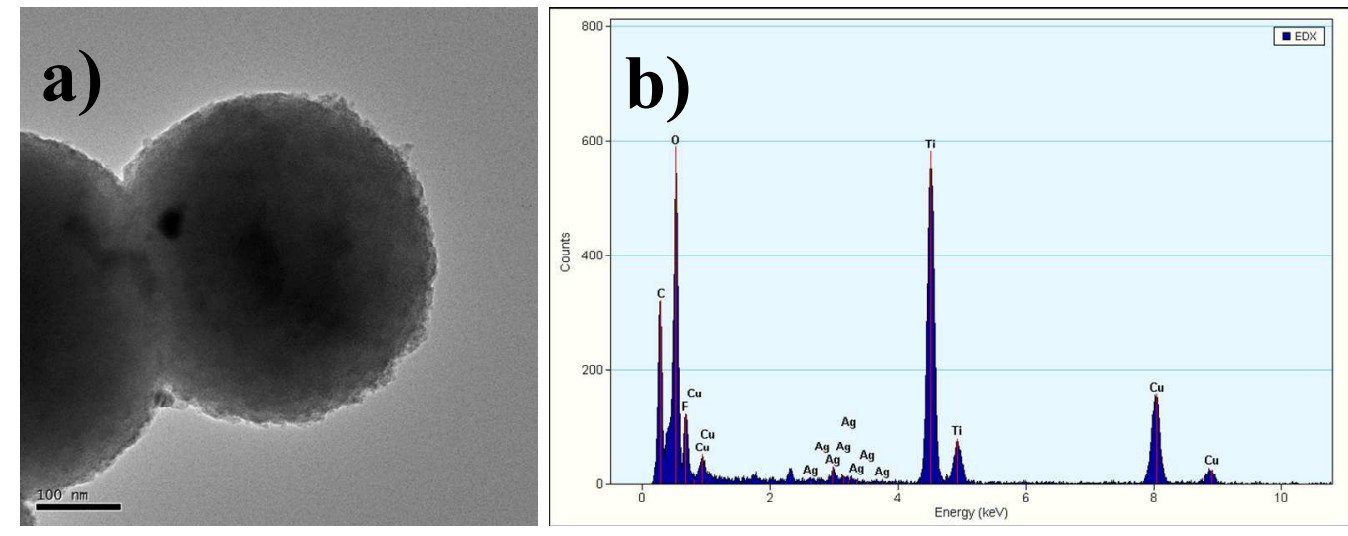

Figure S1. TEM image and EDS curve of the superhydrophobic particles. 


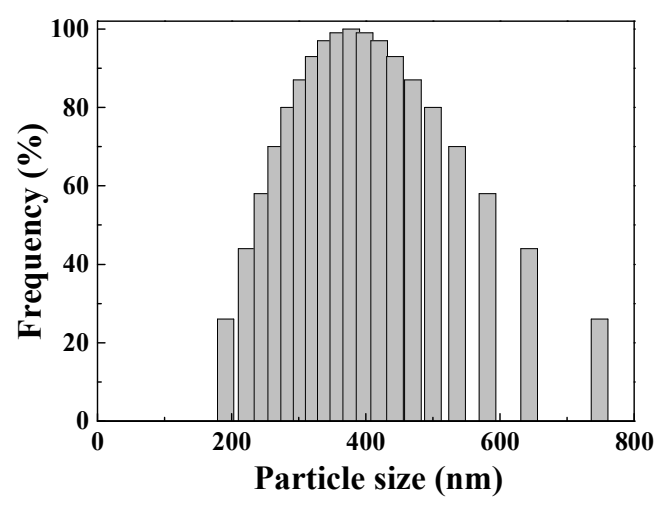

Figure S2. The size distribution of the superhydrophobic titania/polyphenol/Ag particles. 


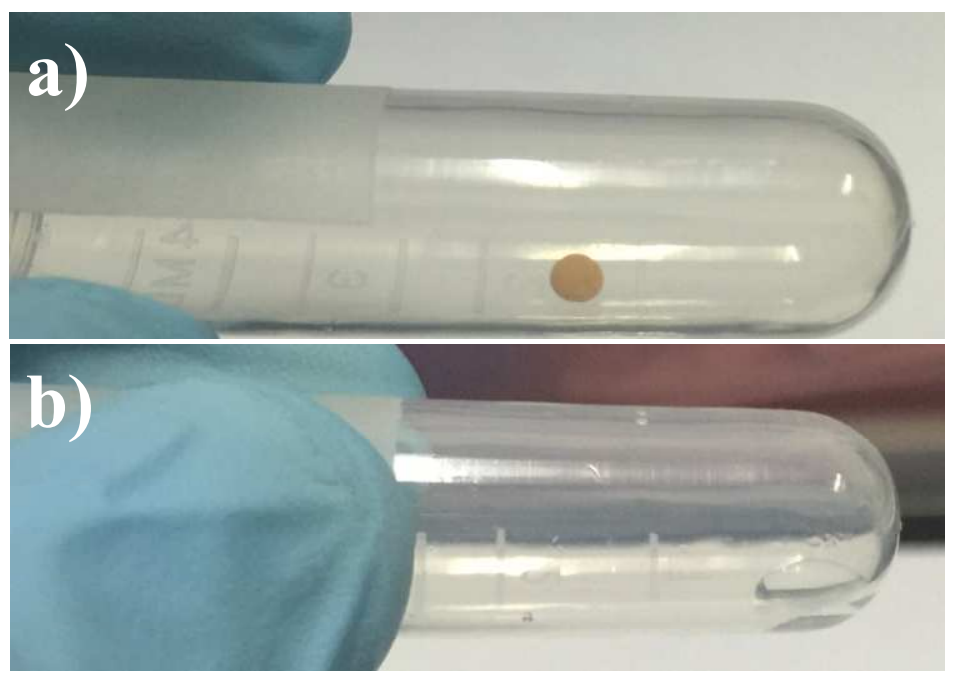

Figure S3. a) Liquid marble and b) water droplet in the internal surface of polypropylene tube. 


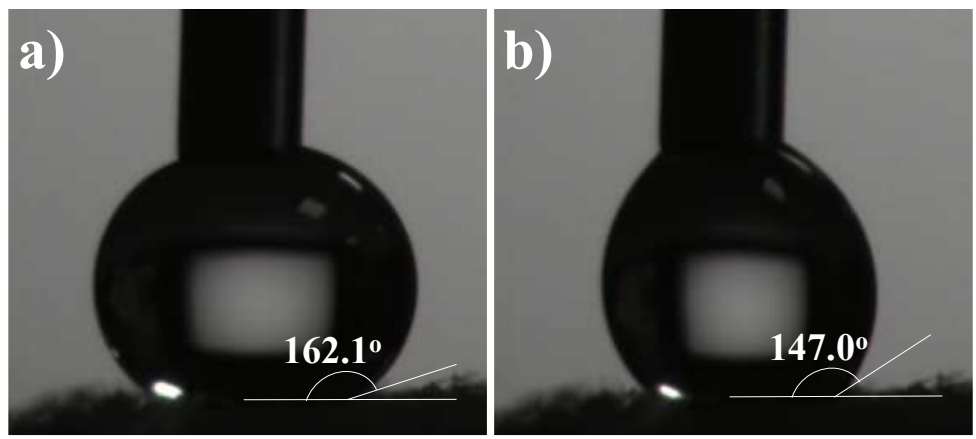

Figure S4. The a) advancing and b) receding water contact angles of the particle-functionalized sponge. 
Two video files:

- Video S1 could be found in the following website:

\section{http://pan.baidu.com/s/1pJPQ6nh}

- Video S2 could be found in the following website:

http://pan.baidu.com/s/1jGUe4ei

- Note: One can download the two video files directly without registration from the above websites or see them online after registration. 